\title{
Correlation between global longitudinal strain and SYNTAX score in coronary artery disease evaluation
}

\author{
Gomaa Abdelrazek*, Alaa Yassin and Khaled Elkhashab
}

\begin{abstract}
Background: Speckle tracking echocardiography may allow the evaluation of myocardial systolic and diastolic dynamics across different physiologic and pathologic conditions beyond traditional echocardiographic techniques. The use of STE longitudinal strain in identification and risk stratification of CAD has good reproducibility and accuracy. The study aims to detect the relationship between SYNTAX score and global longitudinal peak systolic strain (GLPSS) in patients undergoing coronary angiography, with no history of myocardial infarction.

Results: The study included 70 symptomatic patients suspected to have chronic coronary syndrome aging 20-80 years (excluding those with significant structural heart disease). All patients underwent clinical evaluation, surface ECG, laboratory assessment, transthoracic echocardiographic (TTE), color TDI tracings, two-dimensional speckle tracking, and conventional coronary angiography with SYNTAX score calculation. Patients were divided into 3 groups based on the results of the coronary angiogram: normal CAD on angiogram ( $n=10$, control group), low SS $(n=25$, SS $<22)$, and high SS ( $n=35, \mathrm{SS} \geq 22)$.

The mean age was $55 \pm 9.6$ years; $54 \%$ were males; two third of patients were hypertensive; $52 \%$ were diabetic; high percentage of high syntax score were noticed among males, diabetic patients, and smokers; and also low mean of GLS was noticed among diabetic patients and smokers. There was a statistically significant positive correlation between syntax score and each of LVEDD and LVESD and, on the other hand, statistically significant negative correlation between syntax score and each of E/A, GLS, AP2LS, AP3LS, and AP4LS was noticed. Peak GLS cutoff value of 17.8 and 16.5 showed $84 \%$ and $93 \%$ sensitivity and $70 \%$ and $91 \%$ specificity to detect high and low syntax score, respectively.
\end{abstract}

Conclusion: 2D longitudinal strain analysis has incremental diagnostic value over visual assessment during echocardiography in predicting significant coronary artery disease; GLS may offer a potential sensitive tool to detect significant CAD.

Keywords: Chronic coronary syndrome, Two-dimensional speckle tracking, Global longitudinal strain, SYNTAX score

\section{Background}

The diagnosis and assessment of chronic coronary syndrome (CCS) involves clinical evaluation, identifying risk factors for atherosclerosis, and specific cardiac investigations such as different stress testing modalities and coronary imaging [1].

\footnotetext{
* Correspondence: gabrazek@yahoo.com
}

Fayoum University, Fayoum, Egypt
Despite the widespread use of imaging and provocative testing, noninvasive identification of patients with CAD remains a clinical challenge; more than half of the patients had normal or non-obstructive CAD on coronary angiography [2].

The diagnosis of CAD using echocardiography mostly depends on the detection of left ventricle (LV) abnormal wall motion and the assessment of LV ejection fraction (EF). However, there are no abnormal LV wall motions

\section{Springer Open}

(c) The Author(s). 2020 Open Access This article is licensed under a Creative Commons Attribution 4.0 International License, which permits use, sharing, adaptation, distribution and reproduction in any medium or format, as long as you give appropriate credit to the original author(s) and the source, provide a link to the Creative Commons licence, and indicate if changes were made. The images or other third party material in this article are included in the article's Creative Commons licence, unless indicated otherwise in a credit line to the material. If material is not included in the article's Creative Commons licence and your intended use is not permitted by statutory regulation or exceeds the permitted use, you will need to obtain permission directly from the copyright holder. To view a copy of this licence, visit http://creativecommons.org/licenses/by/4.0/. 
at rest in CAD patients without a history of myocardial infarction [3].

The strain values are better than either wall motion or tissue Doppler in the assessment of regional contraction. Also, strain can be used in assessing myocardial viability either at rest or with stress [4].

Significant coronary artery stenosis might cause persistently impaired longitudinal LV function at rest, so 2D-STE is more accurate than conventional 2D echocardiography in evaluating the regional and global myocardial function and assessing infarct size, viability of the infarcted myocardium, and mild changes of myocardial ischemia [5].

Speckle tracking echocardiography is a semiautomated modality so it provides good intraobserver and interobserver reproducibility [6].

The non-invasive quantification of regional myocardial function is an important goal in clinical cardiology. An ultrasonic method that quantifies regional deformation has been introduced based on the principles of "strain" and "strain rate" imaging; it requires a single cardiac cycle for further offline processing and interpretation [7].

STE is a simple, rapid, and accurate method for evaluating the myocardial function, so it is best to assess regional contractile function by measuring peak systolic SR or rate of increase of SR [8].

A good correlation between longitudinal strain and the left ventricular ejection fraction (LVEF) has been shown [8] also; longitudinal strain provides a good quantitative myocardial deformation assessment of each LV segment allowing early detection of systolic dysfunction in patients with preserved LV ejection fraction [9].

The use of STE longitudinal strain can detect and riskstratify CAD with good accuracy and reproducibility. Strain and SR are homogeneously distributed across the myocardium, so mild changes in either measure suggest myocardial dysfunction. Although strain imaging has a potential role in the diagnosis and management of virtually any myocardial disease, its greatest role is in the detection of ischemic heart disease [10].

The Synergy between Percutaneous Coronary Intervention with Taxus and Cardiac Surgery (SYNTAX) score aims to describe coronary anatomy based on anatomic criteria, such as lesion site and complexity; it can predict clinical outcomes after PCI or CABG in multiple vessel disease (MVD) patients and/or left main coronary artery disease [11].

The SYNTAX score involves complex lesions including bifurcations, chronic total occlusions, thrombus, calcification, and small vessel diffuse disease. The score ranges from 0 to greater than 60 in very complex coronary lesions. Patients with SYNTAX scores of 0 to 22 showed comparable outcomes for CABG and PCI while patients with SYNTAX scores greater than 22 CABG patients showed better outcomes than PCI patients. The high SYNTAX scores indicate complex conditions and represent the greatest risks to patients undergoing PCI. The SYNTAX calculator is online at www.syntaxscore. com [12].

The SYNTAX score is the sum of the points of each individual lesion present in the coronary arteries with > $50 \%$ diameter stenosis in vessels $>1.5 \mathrm{~mm}$ diameter. The coronary tree is divided into 16 segments according to the AHA classification. The SYNTAX score algorithm then sums each of these features for a total SYNTAX score. A computer algorithm is then queried and the score value is produced [13]. The SYNTAX II score integrates two anatomic and clinical variables to predict 4year mortality after CABG or PCI. It can accurately predict cardiac mortality and major adverse cardiac events in patients with multivessel specifically unprotected left main coronary disease undergoing PCI [14].

\section{Methods}

This is a prospective cross-sectional study. It was conducted on 70 patients complaining of chest pain who presented to the cardiology outpatient clinic for evaluation of myocardial ischemia.

Enrollment of patients commenced in June 2018 and was completed in April 2019.

Symptomatic patients suspected to have stable coronary artery disease aging $20-80$ were included in the study.

\section{Exclusion criteria}

- Patients with acute myocardial infarction or highrisk unstable angina.

- Patients with a history of previous myocardial infarction or previous recent coronary revascularization.

- Patients with resting regional wall motion abnormalities in echocardiography

- Patients with structural heart disease, e.g., significant left ventricular hypertrophy.

- Patients with more than mild valvular lesions.

- Patients with suboptimal echogenic window.

- Patients with persistent arrhythmia or frequent extra systoles.

Each patient included in the study has been subjected to the following:

- Careful history taking and thorough physical examination.

- 12-lead surface ECG. 
- Transthoracic echocardiography (TTE): Conventional 2D echocardiography was done for each patient then offline 2D-STE analysis was done for the digitally stored images to obtain the longitudinal deformation parameters at rest. Examination was done by using an ultrasound system (Philips EPIQ 7), with a frequency of $1.5-4.3 \mathrm{MHz}$ transducer and high frame rate (60-90 frames/s); LV volumes were traced manually at end-diastole and endsystole in apical four- and two-chamber views using the modified biplane Simpson's method. The mitral inflow velocity curves were measured using pulsedwave Doppler in the apical position at the tips of the mitral valve leaflets, and the E/A-ratio was calculated. Peak velocity of early (E) and atrial (A) diastolic filling and deceleration time of the E-wave (DT) were measured. Color TDI tracings were used to measure the $\mathrm{E} / \mathrm{e}^{\prime}$ after measuring the peak longitudinal early diastolic ( $\left.e^{\prime}\right)$ velocity from the average of the lateral and septal velocities.

- Two-dimensional speckle tracking echocardiography: Three standard apical views were obtained at rest according to the American Society of Echocardiography recommendation. Gain, depth, and sector width were adjusted properly in order to set the frame rate 60 to 80 frames/s. Well-defined cardiac cycle was acquired for each view and stored digitally for offline analysis using the Cardiac Motion Quantification (CMQ) feature on the Q lab 9 software (Philips ultrasound). The end-systole was defined first by defining the aortic valve closure (AVC) as seen in the apical long-axis view. Then, the region of interest (ROI) was set by identifying three points at the end-diastolic frame; 2 annular points low and inside the myocardium at the level of insertion of the mitral valve and 1 apical point at the endocardial border of the apex. The software automatically tracks the internal (endocardial) border and external (epicardial) border in the subsequent frames in $30 \mathrm{~s}$. Each ROI is divided equally by the software into three segments (basal, mid, and apical), in addition to a shared apical cap. Tracking was accepted or rejected and manually corrected by the operator when needed. Numerical and graphical displays of the longitudinal strain were automatically generated for all segments from each view. The same procedure was followed with the apical 4- and apical 2chamber views, using the same sequence. Finally, after completion of the analysis of the three apical views, the software generates a 17 segment bull's eye display of the peak longitudinal systolic strain with automatic calculation of the mean global longitudinal strain (GLS).
- Coronary angiography: All patients underwent coronary angiography on a Siemens Axiom Artis coronary angiographic system (Siemens, Erlangen, Germany) within 1 month of the echocardiography. Selective left and right coronary angiography in multiple views in order to avoid side branch overlaps and foreshortening of the relevant coronary stenosis.

- SYNTAX I score calculation: All images were evaluated by an experienced operator who was blinded to the results of STE. The SYNTAX score was derived from the summation of the individual scores for each separate lesion defined as $\geq 50 \%$ luminal obstruction in vessels $\geq 1.5 \mathrm{~mm}$. The SYNT AX scores were calculated for all patients using dedicated software (available at http://www. syntaxscore.com/calc/start.htm). Thereafter, the patients were divided into 3 groups based on the presence and/or the severity of coronary artery disease (CAD): no CAD on angiogram $(n=10$, control group), low SS ( $n=25, \mathrm{SS}<22)$, and high SS $(n=35$, SS $\geq 22)$.

\section{Statistical analysis}

The collected data were organized, tabulated, and statistically analyzed using SPSS software statistical computer package version 18 (SPSS Inc., USA). For quantitative data, the mean and standard deviation (SD were calculated. Independent $t$ test or one-way ANOVA was used in comparing between any two groups or three groups, respectively. Qualitative data were presented as number and percentages, and chi-square $\left(\chi^{2}\right)$ was used as a test of significance. Pearson's correlation was run to identify the relation of SYNTAX score and GLS with study parameters. The receive operating characteristic (ROC) curve was used to determine the discrimination value of GLS for low and high SYNTAX score and to define optimal cut-points for sensitivity and specificity. For the interpretation of the results of tests of significance, significance was adopted at $p \leq 0.05$.

\section{Ethics approval and consent to participate}

The study was approved by our Institutional ethics committee and an informed written consent was obtained from each patient according to the rules of the Local Ethical Committee of our Faculty (committee reference number: not applicable).

\section{Results}

There were no significant statistical differences between CAD and control groups as regard age, sex, DM, HTN, dyslipidemia, smoking, LVEDD, LVESD, and EF $(p>$ 0.05). 
The CAD group has significantly lower AP4 longitudinal strain compared to the control group (13.9 \pm 3.5 vs. $19.7 \pm 2.9, p<0.001$ ), lower AP3 longitudinal strain compared to the control group ( $14.3 \pm 3.4$ vs. $18.2 \pm 2.8$, $p<0.001)$, lower AP2 longitudinal strain compared to the control group $(14.1 \pm 4$ vs. $17.7 \pm 3.3, p<0.01)$, and lower global longitudinal strain compared to the control group $(14.03 \pm 2.3$ vs. $18.5 \pm 1.4, p<0.001)$ (Table 1$)$.

Higher SYNTAX score significantly correlated with LVEDD and LVESD $(p<0.001)$, E/A ratio $(p<0.04)$, AP2L strain, AP3L strain, AP4L strain, and GLS $(p<$ 0.001) (Table 1).

GLS significantly correlated with serum creatinine ( $p=$ $0.03), \operatorname{LVEDD} 9(p=0.02), \operatorname{LVESD}(p=0.004), \mathrm{E} / \mathrm{A}$ ratio $(p=0.008)$, AP2L strain, AP3L strain, AP4L strain, GLS, and SYNTAX score $(p<0.001)$ (Table 2).

Using GLS cutoff value of $17.8 \%$ predicted low SYNT AX score with $84 \%$ sensitivity and $70 \%$ specificity (Fig. 1). On the other hand, using GLS cutoff value of $15.9 \%$ predicted high SYNTAX score with 97.1\% sensitivity and $90 \%$ specificity (Fig. 2).

\section{Discussion}

Echocardiography is an important cardiac imaging tool in patients with suspected cardiac disease. However, conventional echocardiography has a limited value in the diagnosis and risk stratification of suspected CAD patients as most of these patients with no previous myocardial infarction or myocardial stunning have no wall

Table 1 Comparisons of SYNTAX score categories with echo findings

\begin{tabular}{lllll}
\hline Variables & Normal $(n=10)$ & Low $(n=25)$ & High $(n=35)$ & $p$ value \\
& Mean \pm SD & Mean \pm SD & Mean \pm SD & \\
\hline Age (years) & $51.4 \pm 6.2$ & $58.9 \pm 9.8$ & $54.2 \pm 9.6$ & 0.06 \\
LVEDD & $41.7 \pm 6.1$ & $41.4 \pm 5.3$ & $\mathbf{4 7 . 6} \pm \mathbf{5 . 6}$ & $<\mathbf{0 . 0 0 1}$ \\
LVESD & $28.2 \pm 5.1$ & $28.04 \pm 3.2$ & $\mathbf{3 3 . 2} \pm \mathbf{4 . 8}$ & $<\mathbf{0 . 0 0 1}$ \\
EF & $61 \pm 5.6$ & $60.5 \pm 5.01$ & $58.4 \pm 4.3$ & 0.1 \\
IVS & $0.93 \pm 0.13$ & $0.91 \pm 0.12$ & $0.95 \pm 0.09$ & 0.2 \\
PW & $0.92 \pm 0.12$ & $0.92 \pm 0.012$ & $0.96 \pm 0.09$ & 0.2 \\
E/A & $1.1 \pm 0.33$ & $0.84 \pm 0.29$ & $\mathbf{0 . 8 2} \pm \mathbf{0 . 2 3}$ & $\mathbf{0 . 0 4}$ \\
DT & $157.9 \pm 21.8$ & $140.1 \pm 25.5$ & $146.4 \pm 28.2$ & 0.2 \\
E/e & $8 \pm 0.67$ & $8.5 \pm 1.1$ & $8.2 \pm 1.4$ & 0.5 \\
GLS & $18.5 \pm 1.4$ & $15.7 \pm 2.1$ & $\mathbf{1 2 . 9} \pm \mathbf{1 . 6}$ & $<\mathbf{0 . 0 0 1}$ \\
AP2LS & $17.6 \pm 3.3$ & $16.6 \pm 3.6$ & $\mathbf{1 2 . 3} \pm \mathbf{3 . 2}$ & $<\mathbf{0 . 0 0 1}$ \\
AP3LS & $18.2 \pm 2.8$ & $15.4 \pm 4.04$ & $\mathbf{1 3 . 4} \pm \mathbf{2 . 7}$ & $<\mathbf{0 . 0 0 1}$ \\
AP4LS & $19.7 \pm 2.9$ & $15.4 \pm 3.8$ & $\mathbf{1 2 . 9} \pm \mathbf{2 . 9}$ & $<\mathbf{0 . 0 0 1}$ \\
Creatinine & $1.02 \pm 0.3$ & $1 \pm 0.2$ & $1.1 \pm 0.3$ & 0.5 \\
\hline
\end{tabular}

LVEDD left ventricle end-diastolic dimension, $L V E S D$ left ventricle end-systolic dimension, EF ejection fraction, IVS inter-ventricular septum, $P W$ posterior wall, $D T$ deceleration time, GLS global longitudinal strain, AP2LS apical two longitudinal strain, AP3LS apical three longitudinal strain, AP4LS apical four longitudinal strain
Table 2 Correlation between SYNTAX score and GLS with all study variables among study groups

\begin{tabular}{lll}
\hline Variables & \multicolumn{1}{l}{ GLS } & \\
\cline { 2 - 3 } & $r$ & $p$ value \\
\hline Age (years) & 0.02 & 0.9 \\
LVEDD & $-\mathbf{0 . 2 8}$ & $\mathbf{0 . 0 2}$ \\
LVESD & $-\mathbf{0 . 3 4}$ & $\mathbf{0 . 0 0 4}$ \\
EF & $\mathbf{0 . 2 5}$ & $\mathbf{0 . 0 4}$ \\
IVS & -0.006 & 0.9 \\
PW & -0.07 & 0.6 \\
E/A & $\mathbf{0 . 3 2}$ & $\mathbf{0 . 0 0 8}$ \\
DT & 0.08 & 0.5 \\
E/e & -0.07 & 0.6 \\
AP2LS & $\mathbf{0 . 7 2}$ & $\mathbf{0 . 0 0 1}$ \\
AP3LS & $\mathbf{0 . 6 8}$ & $\mathbf{0 . 0 0 1}$ \\
AP4LS & $\mathbf{0 . 7 2}$ & $\mathbf{0 . 0 0 1}$ \\
Creatinine & $-\mathbf{0 . 2 6}$ & $\mathbf{0 . 0 3}$ \\
SYNTAX score & $-\mathbf{0 . 8 3}$ & $\mathbf{0 . 0 0 1}$
\end{tabular}

LVEDD left ventricle end-diastolic dimension, LVESD left ventricle end-systolic dimension, $E F$ ejection fraction, IVS inter-ventricular septum, $P W$ posterior wall, $D T$ deceleration time, GLS global longitudinal strain, AP2LS apical two longitudinal strain, $A P 3 L S$ apical three longitudinal strain, $A P 4 L S$ apical four longitudinal strain

motion abnormalities at rest. So, finding another resting module to diagnose and predict the severity of CAD would be very beneficial [15].

Speckle tracking echocardiography (STE) is a semiautomated software that allows fast, quantitative, and angle-independent assessment of the three components of myocardial deformation, with great feasibility and reproducibility particularly of the longitudinal one. Several clinical studies confirmed the feasibility of STE-derived longitudinal strain analysis as an adjunctive method for CAD detection [16].

In this prospective study, we studied 70 patients aged 20-80 and collected from an outpatient cardiology clinic; echocardiography (including both conventional and STE) and coronary angiography were done for each patient, and then data was collected and analyzed.

We could identify a cutoff value of GLS with high sensitivity and specificity to detect a high SYNTAX score.

Biering-Sørensen et al. [17], Gaibazzi et al. [18], and Billehaug et al. [19] showed that GLS is significantly lower in patients with obstructive CAD (at least one stenosis $>50 \%$ or $\geq 70 \%$ luminal area reduction) when compared with patients with non-obstructive CAD, and our study showed similar results. And they reported that GLS values at rest have moderate diagnostic accuracy in predicting significant CAD while in our study, it had high diagnostic accuracy, as our results revealed that GLS $\leqslant 16.5$ can predict significant coronary artery stenosis, with sensitivity $93 \%$ and specificity $91 \%$, while 


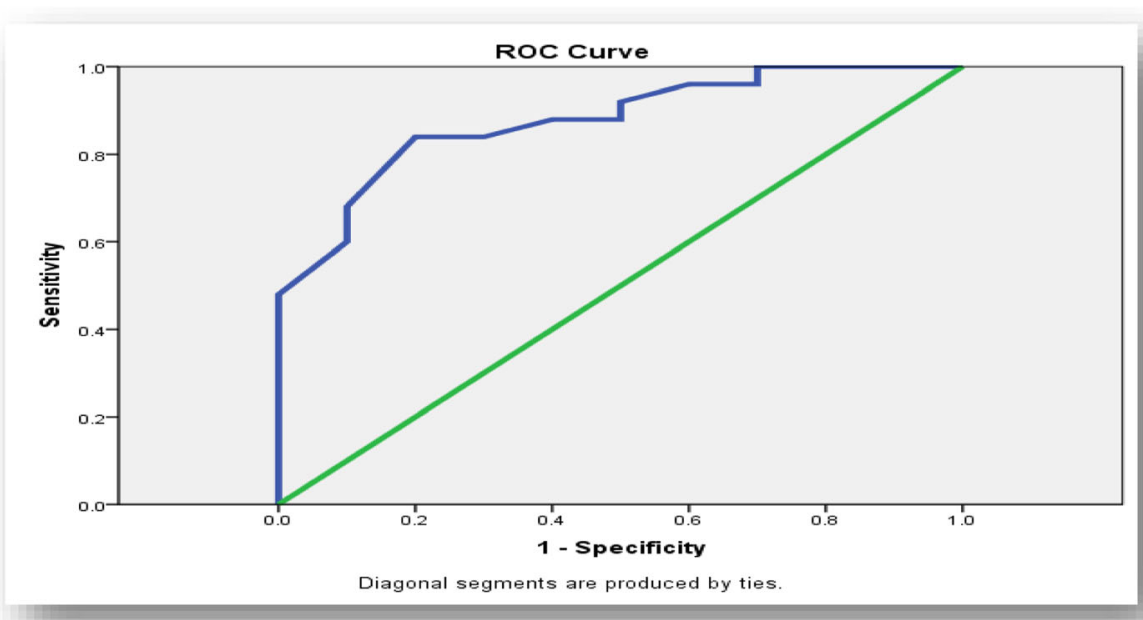

Fig. 1 ROC curve for low SYNTAX score

Biering-Sørensen et al. [17] showed that GLS $\leqslant-18.4 \%$ can predict significant coronary stenosis $(>70 \%)$, with sensitivity $74 \%$ and specificity $58 \%$. Similarly, Gaibazzi et al. [18] showed that GLS $\leqslant-20.7$ may predict significant coronary stenosis (> 50\%), with sensitivity $81.6 \%$ and specificity $84.9 \%$.

In Billehaug et al. [19], GLS measurements have moderate diagnostic accuracy in predicting significant $C A D$ in patients presenting with chest pain. They showed that GLS cutoff value for prediction of CAD varied between - 17.4 and $-19.7 \%$ with sensitivity from 51 to $81 \%$ and specificity between 58 and $81 \%$. The effect of diastolic function and afterload on GLS may explain this finding.

Our study showed that GLS decreased incrementally with increasing SYNTAX score which indicates increasing severity of CAD. Vrettos et al. [20] showed similar results when they studied 71 patients and reported that GLS values were inversely correlated to SYNTAX score values. And they showed that the GLS optimal cutoff value to detect patients with high SYNT AX score was -13.95 (sensitivity $=71 \%$, specificity $=$ $90 \%, p<0.001)$.

There was a positive correlation between high SYNT AX score and being diabetic $(p=0.007)$; this was also noted in Srinivasan et al. [21] where they observed that patients with 5-10 years of diabetes mellitus have a significant increase in the mean SYNTAX score $(p=0.019)$ when compared to those with less diabetes duration.

Another positive correlation was present between SYNTAX score and smoking $(p=0.001)$; this was found

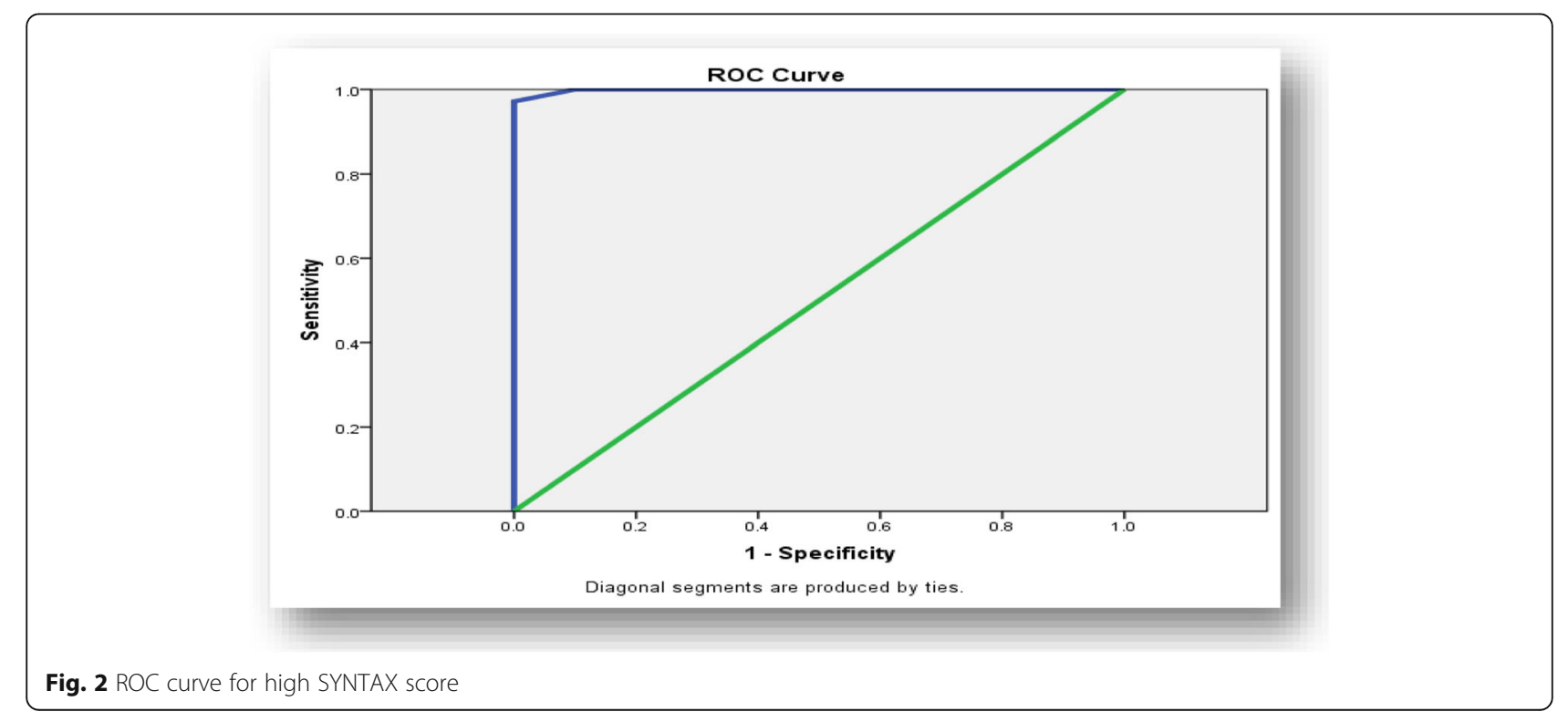


in several studies, among them El Kersh et al. [22], where a statistically significant correlation between age, hypertension, diabetes mellitus, dyslipidemia, and smoking with SYNTAX score was noticed $(p<0.05)$.

El-Sayed et al. [23] found that high SYNTAX score patients have higher E/A ratio and lower deceleration time (DT) when compared with the low SYNTAX score patients ( $p=0.016$ and $p=0.046$, respectively) while our study did not reach these results as the E/A ratio was lower in the high SYNTAX group $(p=0.04)$.

In our study, mean GLS was lower in diabetic patients $13.7 \pm 2.4$ than non-diabetics $15.8 \pm 2.7(p=0.001)$, and this was similar to the results of Elgohary et al. [24] who compared results of patients with controlled and uncontrolled diabetes and found a significant statistical difference in GLS, age, diabetic duration, 2HPP blood sugar level, and E/é ratio in patients with controlled DM compared to uncontrolled DM. Another study by Wierzbowska-Drabik et al. [25] showed that during DSE global and regional LV peak, systolic longitudinal strain revealed lower values in DM patients when compared to non-diabetics: $14.5 \pm 3.6 \%$ vs. $17.4 \pm 4.0 \%$ at rest; $p=$ 0.0001 .

On the other hand, while we found that smokers had significantly lower mean GLS $(p=0.002)$, Farsalinos et al. [26] showed no significant changes in GLS were reported when they studied myocardial function in young and healthy heavy smokers.

Our study results showed a good correlation between left ventricle EF and GLS $(r=0.25 ; p=0.04)$, and Benyounes et al. [27] reported similar results when they approved that two-dimensional GLS can predict LVEF ( $r$ $=-0.53 ; p<0.001)$. Also, Lima et al. [28] concluded that left ventricle EF and GLS showed a powerful correlation $\left(r=0.95 ; r^{2}=0.89 ; p<0.001\right)$, especially in patients with LV systolic dysfunction than those with normal LVEF.

\section{Conclusion}

- Normal left ventricular wall motion at rest cannot exclude significant CAD.

- GLS has a significant correlation with the complexity of CAD as quantified by the widely used scoring system, SYNTAX score.

- Resting 2D-STE-derived global longitudinal strain (GLS) can predict significant CAD with high sensitivity of 84 and $93 \%$ for the detection of low and high SYNTAX score, respectively.

\section{Clinical applications and recommendations}

The present study recommends that adding STE to the standard echocardiography protocol for evaluation of myocardial ischemia in patients with chronic coronary artery disease and using the found cutoff values of GLS can predict patients likely to have more severe and complex CAD and might benefit from invasive coronary angiography and revascularization, but these values should be tested in larger population studies.

\section{Limitations}

- Relatively low number of studied patients and single-center study.

- Excluding patients with wall motion abnormalities at baseline echocardiogram.

- Strain and strain rate are influenced by left ventricular mass, various hemodynamic variables, and software platform type, in addition to myocardial ischemia. All these factors could be potential confounders.

\section{Abbreviations}

2D-STE: Two-dimensional speckle tracking echocardiography; AHA: American Heart Association; AP2LS: Apical two longitudinal strain; AP3LS: Apical three longitudinal strain; AP4LS: Apical four longitudinal strain; CABG: Coronary artery bypass grafting; CAD: Coronary artery disease; DM: Diabetes mellitus; DSE: Dobutamine stress echocardiography; DT: Deceleration time;

ECG: Electrocardiogram; EF: Ejection fraction; ESC: European Society of Cardiology; GLPSS: Global longitudinal peak systolic strain; GLS: Global longitudinal strain; HTN: Hypertension; IHD: Ischemic heart disease; IVS: Interventricular septum; LBBB: Left bundle branch block; LV: Left ventricle; LVEDD: Left ventricle end-diastolic dimension; LVEF: Left ventricular ejection fraction; LVESD: Left ventricle end-systolic dimension; LVH: Left ventricular hypertrophy; MI: Myocardial infarction; PCl: Percutaneous coronary intervention; PW: Posterior wall; PVD: Peripheral vascular disease; ROC: Receiver operating characteristic; ROI: Region of interest; SD: Standard deviation; SPSS: Statistical Package of Social Science; SR: Strain rate; TDI: Tissue Doppler imaging; WMA: Wall motion abnormalities

\section{Acknowledgements}

Not applicable

\section{Authors' contributions}

AY was involved in collecting and analyzing the data. GA was involved in analyzing the data and in preparing the manuscript. KE was involved in analyzing the data. The authors read and approved the final manuscript.

Funding

Not applicable

\section{Availability of data and materials}

The data sets used and/or analyzed during the current study available from the corresponding author on reasonable request.

\section{Competing interest}

The authors declare that they have no conflict of interests.

Ethics approval and consent to participate

The study was approved by the ethical review committee of the Faculty of Medicine, Fayoum University, Egypt, in February 2018 and an informed written consent was obtained from each patient according to the declaration of Helsinki and the rules of the Local Ethics Committee (committee reference number: not applicable).

Consent for publication

Not applicable 
Received: 19 March 2020 Accepted: 3 May 2020

Published online: 15 May 2020

\section{References}

1. Bösner S, Haasenritter J, Becker A, Karatolios K, Vaucher P, Gencer B, Herzig L, Heinzel-Gutenbrunner M, Schaefer JR, Abu Hani M, Keller H, Sönnichsen AC, Baum E, Donner-Banzhoff N (2010) Ruling out coronary artery disease in primary care: development and validation of a simple prediction rule. CMAJ. 182:1295-1300

2. Patel MR, Peterson ED, Dai D, Brennan JM, Redberg RF, Anderson HV, Brindis RG, Douglas PS (2010) Low diagnostic yield of elective coronary angiography. N Engl J Med 362:886-895

3. Elhendy A, van Domburg RT, Bax JJ, Roelandt JR (2000) Significance of resting wall motion abnormalities in 2-dimensional echocardiography in patients without previous myocardial infarction referred for pharmacologic stress testing. J Am Soc Echocardiogr 13(1):1-8

4. Bansal M, Jeffriess L, Leano R, Mundy J, Marwick TH (2010) Assessment of myocardial viability at dobutamine echocardiography by deformation analysis using tissue velocity and speckle-tracking. JACC Cardiovasc Imaging 3(2):121-131

5. Montgomery DE, Puthumana JJ, Fox JM, Ogunyankin KO (2012) Global longitudinal strain aids the detection of non-obstructive coronary artery disease in the resting echocardiogram. Eur Heart J Cardiovasc Imaging 13: 579-587

6. Park CM, March K, Williams S et al (2013) Feasibility and reproducibility of left ventricular rotation by speckle tracking echocardiography in elderly individuals and the impact of different software. PLoS One. 8(9):e75098

7. Perk G, Tunick PA, Kronzon I (2007) Non-Doppler two-dimensional strain imaging by echocardiography--from technical considerations to clinical applications. J Am Soc Echocardiogr. 20(3):234-243

8. Witkowski TG, Thomas JD, Delgado $V$ et al (2012) Changes in left ventricular function after mitral valve repair for severe organic mitral regurgitation. Ann Thorac Surg. 93(3):754-760

9. Shivu GN, Abozguia K, Phan TT et al (2009) Increased left ventricular torsion in uncomplicated type 1 diabetic patients: the role of coronary microvascular function. Diabetes Care. 32(9):1710-1712

10. Jamal F, Kukulski T, Sutherland GR et al (2002) Can changes in systolic longitudinal deformation quantify regional myocardial function after an acute infarction? An ultrasonic strain rate and strain study. J Am Soc Echocardiogr. 15(7):723-730

11. Sianos G, Morel MA, Kappetein AP, Morice MC, Colombo A, Dawkins K et al (2005) The SYNTAX score: an angiographic tool grading the complexity of coronary artery disease. Eurolntervention 1:219-227

12. Valgimigli M, Serruys PW, Tsuchida K et al (2007) Cyphering the complexity of coronary artery disease using the syntax score to predict clinical outcome in patients with three-vessel lumen obstruction undergoing percutaneous coronary intervention. Am J Cardiol 99(8):1072-1081

13. Serruys PW, Onuma Y, Garg S, Sarno G, van den Brand M, Kappetein AP et al (2009) Assessment of the SYNTAX score in the Syntax study. Eurolntervention 5:50-56

14. Farooq V, van Klaveren D, Steyerberg EW et al (2013) Anatomical and clinical characteristics to guide decision making between coronary artery bypass surgery and percutaneous coronary intervention for individual patients: development and validation of SYNTAX score II. Lancet. 381:639650

15. Rumbinaitè E, Žaliaduonytė-Pekšienè D, Vieželis M et al (2016) Dobutaminestress echocardiography speckle-tracking imaging in the assessment of hemodynamic significance of coronary artery stenosis in patients with moderate and high probability of coronary artery disease. Medicina. 52:331339

16. $\mathrm{Ng} \mathrm{A}$, Sitgres M, Phoung N et al (2009) Incremental value of 2-dimensional speckle tracking strain imaging to wall motion analysis for detection of coronary artery disease. Am heart J 158:836-844

17. Biering-Sørensen T, Hoffman S, Mogelvang R (2014) Myocardial strain analysis by 2-dimensional speckle tracking echocardiography improves diagnostics of coronary artery stenosis in stable angina pectoris. Circ Cardiovasc Imag. 7:58-65

18. Gaibazzi N, Pigazzani F, Reverberi C, Porter TR (2014) Rest global longitudinal 2D strain to detect coronary artery disease in patients undergoing stress echocardiography: a comparison with wall-motion and coronary flow reserve responses. Echo Res Pract:61-70
19. Billehaug N, Vidar R, Edvardsen T (2015) Diagnostic accuracy of left ventricular longitudinal function by speckle tracking echocardiography to predict significant coronary artery stenosis. BMC Med Ima. 15:25

20. Vrettos A, Dawson D, Grigoratos CN, Nihoyannopoulos P (2016) Correlation between global longitudinal peak systolic strain and coronary artery disease severity as assessed by the angiographically derived SYNTAX score. Echo Res Pract 3:29-34

21. Srinivasan $M$, Kamath $P$, Bhat $N$ (2016) Severity of coronary artery disease in type 2 diabetes mellitus: does the timing matter? Indian Heart J. 68(2):158163

22. El Kersh AM, Reda AA, El Hadad MG, El-Sharnouby KH (2018) Correlation between SYNTAX score and pattern of risk factors in patients referred for coronary angiography in Cardiology Department, Menoufia University. World Journal of Cardiovascular Diseases 8:431-439

23. El-Sayed I, Attia M, Mosaad M (2017) Correlation between the degree of diastolic function and severity of coronary artery disease as defined by SYNT AX score. N Y Sci J 10(1):13-23

24. Elgohary AA, Shalaby MA, Mahfouz RA, Mohamed MG (2017) Effect of diabetic duration on left ventricular global longitudinal strain by speckle tracking imaging. Am J Cardiol 119(8):e6

25. Wierzbowska-Drabik K, Trzos E, Kurpesa M et al (2017) Diabetes as an independent predictor of left ventricular longitudinal strain reduction at rest and during dobutamine stress test in patients with significant coronary artery disease. Eur Heart J Cardiovasc Imaging 90:1-11

26. Farsalinos K, Tsiapras D, Kyrzopoulos S et al (2013) Acute and chronic effects of smoking on myocardial function in healthy heavy smokers: a study of Doppler flow, Doppler tissue velocity, and two dimensional speckle tracking echocardiography. Echocardiography 30:285-292

27. Benyounes N, Lang S, Soulat-Dufour L, Obadia M, Gout O, Chevalier G et al (2015) Can global longitudinal strain predict reduced left ventricular ejection fraction in daily echocardiographic practice? Arch Cardiovasc Dis 108:50-56

28. Lima MSM et al (2017) Global longitudinal strain or left ventricular twist and torsion? Which correlates best with ejection fraction? Arquivos Brasileiros de Cardiologia 109(1):23-29

\section{Publisher's Note}

Springer Nature remains neutral with regard to jurisdictional claims in published maps and institutional affiliations.

\section{Submit your manuscript to a SpringerOpen ${ }^{\circ}$ journal and benefit from:}

- Convenient online submission

- Rigorous peer review

- Open access: articles freely available online

- High visibility within the field

- Retaining the copyright to your article

Submit your next manuscript at $>$ springeropen.com 